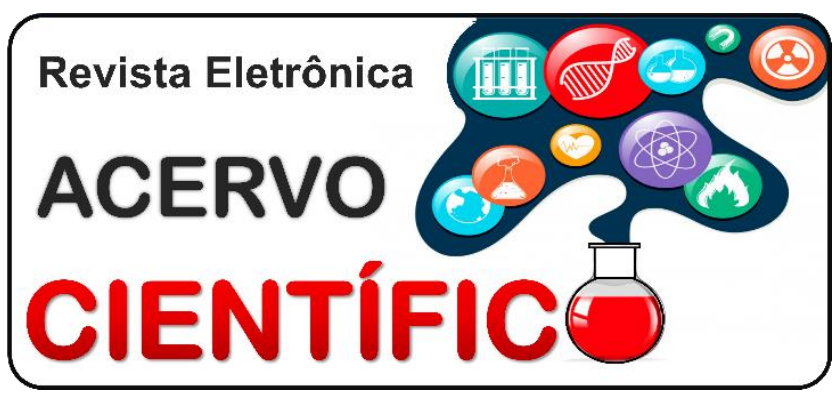

\section{REVISÃO BIBLIOGRÁFICA}

Recebido em: $1 / 2021$

Aceito em: $1 / 2021$

Publicado em: 3/2021

\title{
Cirurgia minimamente invasiva versus craniectomia no tratamento da hipertensão intracraniana
}

\author{
Minimally invasive surgery versus craniectomy in the treatment of intracranial hypertension \\ Cirugía mínimamente invasiva versus craniectomía en el tratamiento de la hipertensión \\ intracraneal
}

Ian Xavier Paschoeto dos Santos ${ }^{1 *}$, Aline Trovão Queiroz ${ }^{2}$, Amós Matos Moreira Dias ${ }^{3}$, Felipe Martins Costa ${ }^{4}$, Isabella Lidório Pires Silva ${ }^{5}$, Luiz Eduardo da Cunha Lima Diaz ${ }^{6}$, Maria Eduarda Santos Ritt ${ }^{7}$, Mariana Vanon Moreira ${ }^{8}$, Milla Giancristofaro Dutra ${ }^{8}$, Octávio Augusto Soares Dias ${ }^{8}$.

\begin{abstract}
Resumo: O presente artigo apresenta uma revisão da Craniectomia (CT) e da Cirurgia Minimamente Invasiva (CMI), com o intuito de comparar a valência dessas técnicas no tratamento da Hipertensão Intracraniana (HIC), visto que a alta pressão intracraniana pode gerar déficits irreversíveis, como a herniação cerebral. Por intermédio da exposição de categorias e características, tanto da CT quanto da CMI, buscou-se avaliar a melhor conjuntura de aplicação de determinado método. O estudo esclarece a metodologia da CMI pela Remoção Neuroendoscópica e Aspiração Estereotáxica, assim como suas limitações e benefícios. De forma análoga, descreve-se o mecanismo de CT e discute-se o cenário mais propício para a sua utilização. Por fim, conclui-se, a partir dos dados, que a Cirurgia Minimamente Invasiva é favorável à redução da taxa de ressangramentos, do risco de edemas cerebrais e menor taxa de infecções, porém limitada na visualização necessária da hemostasia e no imediato alívio da pressão intracraniana, enquanto que a CT possibilita consequências indesejáveis e apresenta-se antagônico à homeostasia dos vasos.
\end{abstract}

Palavras-chave: Craniectomia, Hipertensão intracraniana, Procedimentos cirúrgicos minimamente invasivos.

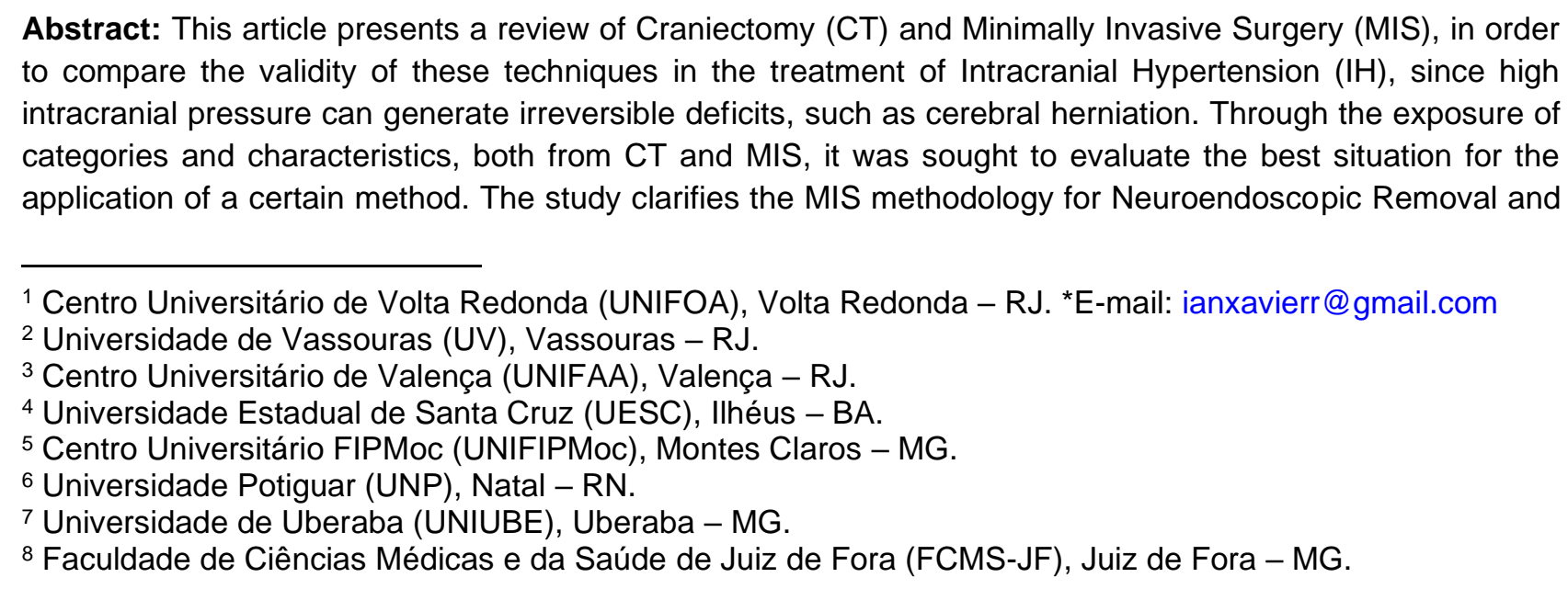

Abstract: This article presents a review of Craniectomy (CT) and Minimally Invasive Surgery (MIS), in order to compare the validity of these techniques in the treatment of Intracranial Hypertension $(\mathrm{IH})$, since high intracranial pressure can generate irreversible deficits, such as cerebral herniation. Through the exposure of categories and characteristics, both from CT and MIS, it was sought to evaluate the best situation for the application of a certain method. The study clarifies the MIS methodology for Neuroendoscopic Removal and

\footnotetext{
${ }^{1}$ Centro Universitário de Volta Redonda (UNIFOA), Volta Redonda - RJ. *E-mail: ianxavierr@gmail.com

2 Universidade de Vassouras (UV), Vassouras - RJ.

${ }^{3}$ Centro Universitário de Valença (UNIFAA), Valença - RJ.

${ }^{4}$ Universidade Estadual de Santa Cruz (UESC), Ilhéus - BA.

${ }^{5}$ Centro Universitário FIPMoc (UNIFIPMoc), Montes Claros - MG.

${ }^{6}$ Universidade Potiguar (UNP), Natal - RN.

7 Universidade de Uberaba (UNIUBE), Uberaba - MG.

${ }^{8}$ Faculdade de Ciências Médicas e da Saúde de Juiz de Fora (FCMS-JF), Juiz de Fora - MG.
} 
Stereotactic Aspiration, as well as its limitations and benefits. Similarly, the CT mechanism is described and the most favorable scenario for its use is discussed. Finally, it is concluded, from the data, that Minimally Invasive Surgery is favorable to the reduction of the bleeding rate, the risk of cerebral edema and lower infection rate, however limited in the necessary visualization of hemostasis and in the immediate relief of pressure intracranial, while CT allows undesirable consequences and is antagonistic to vessel homeostasis.

Key words: Craniectomy, Intracranial hypertension, Minimally invasive surgical procedures.

Resumen: Este artículo presenta una revisión de Craniectomía (CT) y la Cirugía Mínimamente Invasiva (CMI), con el fin de comparar la validez de estas técnicas en el tratamiento de la Hipertensión Intracraneal (HIC), ya que la presión intracraneal alta puede generar déficits irreversibles, tales como hernia cerebral. Mediante la exposición de categorías y características, tanto del CT como del CMI, se intentó evaluar la mejor situación para aplicar un método en particular. El estudio aclara la metodología CMI mediante la Remoción Neuroendoscópica y pasar la Aspiración Estereotáctica, así como sus limitaciones y beneficios. Análogamente, se describe el mecanismo de CT y se discute el escenario más favorable para su uso. Finalmente, se concluye, a partir de los datos, que la Cirugía Mínimamente Invasiva es favorable a la reducción de la tasa de sangrado, el riesgo de edema cerebral y menor tasa de infección, aunque limitada en la visualización necesaria de la hemostasia y en el alivio inmediato de la presión. intracraneal, mientras que la CT permite consecuencias indeseables y es antagónica a la homeostasis vascular.

Palabras clave: Craniectomía, Hipertensión intracraneal, Procedimientos quirúrgicos mínimamente invasivos.

\section{INTRODUÇÃO}

A hipertensão intracraniana (HIC) é uma emergência neurológica indicativa de que os mecanismos adaptativos de complacência intracraniana foram comprometidos. Tal síndrome consiste no aumento da pressão intracraniana (PIC) acima de $22 \mathrm{mmHg}$ por mais de cinco minutos, contrariando as diretrizes clínicas que estipulam, como ideal, valores inferiores a $20 \mathrm{mmHg}$ (KOENIG MA, 2018; DE LIMA OLIVEIRA M, et al., 2018; KASPROWICZ M, et al., 2015). Ademais, como etiologia dessa elevação, pode-se destacar: 1) processos com efeito de massa (como tumores, acidente vascular isquêmico ou hemorrágico); 2) meningite; 3) encefalite; 4) trombose de seio venoso; 5) pseudotumor cerebral (KOENIG MA, 2018). Tais condições possuem como fatores de risco a pré-existência de hipertensão arterial sistêmica ou de angiopatia amilóide cerebral, a idade avançada do indivíduo, o uso crônico de antitrombóticos e a localização de acidente vascular encefálico hemorrágico (HEMPHILL III JC, et al., 2015).

Comumente, os sintomas da HIC consistem na tríade de Cushing, isto é, hipertensão arterial, bradicardia e arritmia respiratória, mas, além disso, podem ser detectados edema de papila (compressão da veia central da retina pelo líquido cefalorraquidiano), cefaleia de forte intensidade, náuseas, vômitos em jato e rebaixamento súbito do nível de consciência (KOENIG MA, 2018). Igualmente, o diagnóstico e o manejo dos pacientes com HIC devem ser rápidos e precisos, visto que a deterioração cerebral é notificada logo nas primeiras horas após o início de sua manifestação em 15-23\% dos casos. Dessa forma, as taxas de morbimortalidade são altas, variando entre $40 \%$ e $50 \%$ e, se a HIC estiver associada à hemorragia intraventricular, essa porcentagem pode chegar até $80 \%$ dos acometidos. Não obstante, entre os sobreviventes, $61 \%$ a $88 \%$ são dependentes de outros indivíduos para atividades de vida diária mesmo 6 meses após a hemorragia. Dessa maneira, o risco de deterioração neurológica precoce e a alta taxa de prognósticos ruins em longo prazo enfatizam a necessidade de tratamento efetivo para tal comorbidade (SCAGGIANTE J, et al., 2018; FIORELLA D, et al., 2016; HEMPHILL III JC, et al., 2015).

Entre as possíveis linhas terapêuticas, destacam-se dois métodos cirúrgicos: a craniectomia (CT) e a cirurgia minimamente invasiva (CMI) cerebral. A CT é um método cirúrgico convencional que consiste na retirada de um flap ósseo com abertura da dura-máter para alívio da hipertensão intracraniana e, assim, diminuição dos efeitos deletérios do edema cerebral (TANG Y, et al., 2019; KOLIAS AG, et al., 2018). Já a 
CMI é uma técnica cirúrgica mais atual que visa tratar a HIC por meio de agressão mínima ao parênquima cerebral, preservando a anatomia encefálica (XIA Z, et al., 2018).

Nessa perspectiva e considerando a relevância do assunto, o presente estudo tem por objetivo a realização de uma revisão narrativa da literatura que visa a comparação da eficácia da CMI frente a da CT no tratamento da HIC. A partir dessa análise, protocolos podem ser melhor estruturados e novos estudos sobre a temática fomentados.

\section{REVISÃO BIBLIOGRÁFICA}

A perfusão sanguínea cerebral ocorre de forma diferente quando comparada ao restante do corpo devido à resistência gerada pela rígida calota craniana. A pressão intracraniana $(P I C)$ é regulada por um sistema de compensação entre o volume sanguíneo, o líquido cefalorraquidiano e o tecido encefálico. No entanto, uma perda desse equilíbrio ocasiona a hipertensão intracraniana (HIC), cujo tratamento ideal é uma descompressão feita em estágios iniciais da comorbidade. Isso ocorre, pois o manejo tardio da PIC pode ocasionar déficits irreversíveis, como a herniação cerebral, a qual consiste no deslocamento de uma parte do encéfalo através de um orifício intracraniano (HEMPHIL JC, et al., 2015). Além disso, uma compressão do tecido cerebral contra a calota craniana pode impedir a passagem de fluxo sanguíneo, ocasionando a isquemia (KASPROWICZ M, et al., 2016).

A maioria dos casos de progressão da HIC para hemorragia encefálica já apresenta, nas primeiras horas de sangramento, um estresse tanto físico quanto psicológico, dor e disfunção do sistema autonômico, sendo também verificada uma expansão do volume do hematoma em até $38 \%$ dos pacientes. Cabe ressaltar que a cada um $\mathrm{cm} 3$ de aumento do hematoma, o risco de morte ou invalidez aumenta em cerca de $7 \%$ dos acometidos (SHI L, et al, 2017). Dessa forma, este hematoma precisa ser rapidamente removido, diminuindo, assim, a hemorragia existente. Logo, evidencia-se que o objetivo principal da operação é justamente aliviar a pressão no tecido cerebral e, com isso, reduzir ao máximo os possíveis danos secundários. Nesse sentido, podem ser citadas duas cirurgias descompressivas passíveis de proporcionar uma resolução no quadro clínico: a cirurgia minimamente invasiva (CMI) cerebral e a craniectomia (CT), devendo-se optar por aquela que reduz ao máximo o acometimento do tecido neuronal (KIM CH, et al., 2019).

A priori, a CMI pode ser realizada através de duas técnicas cirúrgicas: a) remoção neuroendoscópica $(\mathrm{RN})$; b) aspiração estereotáxica (AE). A RN refere-se às neurocirurgias realizadas com o auxílio de um endoscópio, através do qual o cirurgião consegue direcionar seu campo de visão por meio de uma câmera acoplada ao sistema. Além disso, são também utilizados um monitor de alta qualidade e um foco de luz, o que garante nitidez, luminosidade ideal e uma visão privilegiada de dentro do crânio para o cirurgião. Para que a RN seja realizada, primeiramente, aplica-se anestesia geral com incisão de aproximadamente três $\mathrm{cm}$ na pele na localização do hematoma previamente definida por tomografia computadorizada. Em seguida, usa-se uma broca para a penetração no crânio e, com uma agulha de punção lombar, o canal é aberto e uma seringa realiza a sucção do fator causador da PIC, a reduzindo inicialmente. Por último, uma bainha transparente é introduzida no decorrer da agulha de punção e, por fim, é inserido o neuroendoscópio e um cateter metálico de sucção, eliminando completamente o hematoma e, assim, a HIC (XU X, et al., 2018).

Além deste método cirúrgico minimamente invasivo supracitado, há a $A E$, que é um procedimento de alta precisão e com um grau de complexidade bem mais elevado. Nela, usa-se um sistema de coordenadas tridimensionais para localizar pequenos alvos no interior do crânio e, através de uma agulha de precisão milimétrica se é alcançado o alvo tecidual almejado. Para a realização de tal técnica, faz-se necessária a fixação óssea de um arco de estereotaxia e a inserção de um multi cateter - sob anestesia local. Em seguida, drena-se a etiologia da PIC com uma seringa de $10 \mathrm{~mL}$ e injeta-se Uroquinase dissolvida em um $\mathrm{mL}$ de $\mathrm{NaCl}$ 0,9\% (KIM CH, et al., 2019). Isso permite que haja uma resolução da comorbidade exposta, diminuindo a probabilidade de futuros danos secundários, como a herniação craniana.

Dentre os benefícios da $\mathrm{CMI}$, destaca-se principalmente a menor taxa de ressangramento em comparação a todos os outros métodos cirúrgicos para reversão da HIC, inclusive quando comparada à CT. Isso acontece 
porque, na drenagem inicial, o fluxo de aspiração por segundo não é elevado, de forma que o sangramento seja drenado de modo contínuo e lento, promovendo, assim, uma redução gradual da pressão intracavitária, o que facilita a hemostasia do local e reduz a ocorrência de ressangramento (WANG W, et al., 2017). Em contrapartida, na CT o hematoma é removido completamente em uma única abordagem cirúrgica, gerando uma diminuição brusca e abrupta da pressão o que pode gerar um rompimento elevado de vasos sanguíneos, desencadeando frequentemente ressangramentos e isquemia. Com isso, em tal procedimento, pode-se ocorrer novamente o aumento da PIC, piorando o prognóstico do paciente e trazendo a necessidade de realização de novas cirurgias (WANG W, et al., 2017).

Igualmente, diferentemente de outras técnicas cirúrgicas, a CMI não exige uma realocação do parênquima cerebral, o que traz como grande vantagem a diminuição do risco de edemas cerebrais ocasionados pela própria cirurgia. Além disso, a CMI também permite uma escolha mais precisa e segura da rota até um possível local de sangramento, visto que tal método possibilita uma visualização do cirurgião da hemorragia em múltiplos ângulos, minimizando erros durante o processo cirúrgico (NAM TM e KIM YZ, 2019). Ademais, devido a menor incisão na pele e ao menor tempo cirúrgico, um outro ponto positivo da CMl é uma menor taxa de infecções, o que gera melhores prognósticos e uma melhoria na qualidade de vida de pacientes quando comparados àqueles submetidos à outros tipos de procedimentos (TANG Y, et al., 2018; YE Z, et al., 2017).

Dentre os pontos negativos, aponta-se que a CMI é mais adequada para os hematomas de $25 \mathrm{a} 40 \mathrm{ml}$, de forma que se deve optar por outra técnica, como a CT, em casos de volume superiores a estes (YAMASHIRO $\mathrm{S}$, et al., 2015). Ademais, como não há a abertura da calota craniana, a HIC não é aliviada imediatamente durante a cirurgia, de forma que se aumenta o risco de herniação encefálica até que a PIC seja regulada (WANG GQ, et al., 2014). Não obstante, foi verificado que a sucção propiciada pela CMI usualmente não é suficiente para o alívio da HIC em casos de pacientes que desenvolveram hematomas decorrentes de edema ou infarto cerebral, o que faz com que outro tipo de cirurgia, como a própria CT, seja mais recomendada também nesses casos (HEMPHILL III, et al., 2015).

Em outro âmbito, o procedimento de CT é mais convencional e consiste na prática de remoção de uma camada óssea, expondo a dura-máter subjacente. Assim, visa reverter a natureza rígida e não complacente da caixa craniana e dessa meninge, fornecendo espaço a fim de controlar o edema cerebral e o aumento da PIC (KOLIAS AG, et al., 2018; ZHAO XH, et al., 2019). Tal técnica cirúrgica mostra-se eficaz quando realizada precocemente como intervenção primária em pacientes com HIC resultante de traumas cranioencefálicos (os quais necessitam da realização de uma intervenção ainda mais rapidamente e que possibilite um alívio imediato da HIC) ou como uma medida secundária quando as terapias de primeira linha falham (HALEY MD, et al., 2018).

Cabe mencionar que existem dois tipos de CT: a primária e a secundária. Na CT primária, o retalho ósseo da craniotomia não é substituído após a retirada de uma lesão de massa intracraniana. Assim, tal técnica de operação é mais usada após a remoção de um hematoma subdural agudo, já que o edema cerebral acaba impedindo a substituição do retalho ósseo que poderia ser feito no final da cirurgia. Além disso, outro motivo para não haver a substituição desse fragmento craniano é a tentativa de se realizar uma medida preventiva quando há a preocupação da ocorrência de novos edemas no período pós-operatório imediato, o que agrava o quadro clínico do enfermo (MARTIN S, et al., 2017).

Por outro lado, a CT secundária já é mais comumente realizada como uma forma de intervenção de último nível, na qual a HIC é identificada somente após o período de monitorização da PIC. Esta é mais raramente escolhida como método de abordagem, sendo utilizada mais como opção quando há a necessidade de realizar no paciente uma terapia de segundo nível para controlar níveis onde a PIC não se encontra tão elevada, isto é, em casos nos quais a hipertensão intracraniana não atingiu valores substanciais para configurar o quadro clínico, mas que o prognóstico do paciente provavelmente levará a um aumento dessa PIC. Ademais, existem três principais formas de se fazer uma abordagem para a realização da craniectomia descompressiva secundária que são: a) craniectomia bifrontal; b) hemicraniectomia unilateral e, por último, c) hemicraniectomia bilateral (MARTIN S, et al., 2017). 
Dentre os principais pontos positivos da CT, pode-se destacar: 1) uma redução da mortalidade de pacientes com HIC; 2) uma melhor visão do hematoma; 3) uma remoção imediata da parte lesada; 4) uma melhora na circulação sanguínea local; 5) um melhor prognóstico; 6) uma diminuição do tempo dos pacientes internados na UTI; 7) um menor tempo dos acamados na enfermaria (RUBIANO AM, et al., 2019; ZHAO XH, et al., 2019; KOLIAS AG, et al., 2018; KOLIAS AG, et al., 2016; HALEY MD, et al., 2018).

Dessa forma, a CT pode ser utilizada em pacientes comatosos que apresentam um hematoma subdural agudo, assim como em doentes em coma com edema cerebral originado na fase inicial após a lesão no local (KOLIAS AG, et al., 2016). Dentre os malefícios que podem ocorrer com a realização da CT, destacam-se os riscos comuns a todas as cirurgias invasivas, tais como: a possibilidade de se instalar uma infecção, convulsões pós-cirúrgica, dificuldade na cicatrização da ferida, aumento da deterioração neurológica. Além desses supracitados, por ser um procedimento de grande complexidade, a cirurgia é demorada e, consequentemente, há um maior custo operatório, já que é necessário mais anestesia e um maior trabalho da equipe hospitalar. Além disso, existem pontos técnicos que precisam ser mais minimamente detalhados, como por exemplo, o tamanho do flap ósseo a ser removido para que não haja o aumento da PIC ou progressão da hérnia cerebral, o que pode piorar o quadro clínico do paciente enfermo.

Ademais, além da CT, o paciente precisa de um procedimento adicional que é denominado como cranioplastia. Ele consiste em uma técnica cirúrgica que visa reconstruir o defeito iatrogênico do crânio, valendo-se de materiais como titânio, metacrilato de metila, implantes de polieteretercetona, hidroxiapatita, biocerâmica, ou ainda o próprio osso do paciente que foi removido anteriormente (DAVID BK, 2015).

Nesse ínterim, outro equipamento de proteção deve ser usado para evitar mais lesões cerebrais proveniente de quedas inadvertidas ou outros traumas. Outrossim, vale ressaltar que a cranioplastia melhora a perfusão cerebral, dessa forma, deve ser realizada o quanto antes, no prazo de até 12 semanas após a realização da CT. Logo, esse método tem como uma das desvantagens o fato de submeter o paciente a um grande trauma, além de ter um espectro estreito de indicações, mau prognóstico e alto custo; limitando suas aplicações (MARTIN S, et al., 2017; KIM CH et al., 2019).

Além disso, o ressangramento pós-cirúrgico demonstrou ser mais prevalente na CT quando comparado à CMI. Outrossim, a CT pode evoluir com complicações como: um higroma subdural, uma laceração do seio dural, uma infecção do retalho, uma hidrocefalia, possíveis convulsões pós-cirúrgicas, uma perda de líquido cefalorraquidiano, uma reabsorção óssea do retalho, uma síndrome do retalho cutâneo afundado e, por último, uma hérnia paradoxal (MARTIN S, 2017; GENE AG, 2016).

Portanto, nos últimos anos, os estudos sobre a aplicação da evacuação minimamente invasiva do hematoma intracraniano para hemorragia intracerebral hipertensiva têm aumentado, e essas análises mostram que esse método tem grande valor, pois possuem vantagens de operação simples, com trauma mínimo e alta eficiência, além de ser feito em um curto tempo de operação e proporcionar uma maior segurança e confiabilidade tanto para o paciente enfermo quanto para o cirurgião que escolhe tal método de cirurgia. Ademais, tal técnica possui uma capacidade maior de melhorar efetivamente a função nervosa de pacientes que cursavam com uma hemorragia cerebral hipertensiva (Qin ZH, et al. 2013).

\section{CONSIDERAÇÕES FINAIS}

A partir dos dados supracitados, é possível concluir que a CMI, quando comparada à $\mathrm{CT}$, é mais eficaz na redução da PIC. Outrossim, por ser um procedimento de alta precisão e por possibilitar uma visualização do fator etiológico por múltiplos ângulos, nota-se menor taxa de infecções, redução do risco de edemas cerebrais e diminuição da taxa de ressangramentos, gerando melhores prognósticos e melhoria na qualidade de vida dos pacientes. No entanto, cabe ressaltar que a CMI é adequada para hematomas de 25 a $40 \mathrm{ml}$, de forma que se recomenda a CT para casos de volume superiores. Não obstante, a CMI não resulta em um alívio imediato da HIC, aumentando os riscos de herniação encefálica. 


\section{REFERÊNCIAS}

1. FIORELLA D, et al. Minimally invasive surgery for intracerebral hemorrhage rationale, review of existing data and emerging technologies. Stroke, 2016; 47: 1399-1406.

2. GRINDLINGER GA, et al. Decompressive craniectomy for severe traumatic brain injury: clinical study, literature review and meta-analysis. SpringerPlus, 2016; 5(1): 1605.

3. HALEY MD, et al. Retrospective methods analysis of semiautomated intracerebral hemorrhage volume quantification from a selection of the STICH II cohort (early surgery versus initial conservative treatment in patients with spontaneous supratentorial lobar intracerebral haematomas). Stroke, 2018; 49(2): 325-332.

4. HEMPHILL III, et al. Guidelines for the Management of Spontaneous Intracerebral Hemorrhage A Guideline for Healthcare Professionals From the American Heart Association/American Stroke Association. Stroke, 2015; 46: 20322060.

5. KASPROWICZ M, et al. Intracranial pressure, its components and cerebrospinal fluid pressure-volume compensation. Acta Neurologica Scandinavica, 2016; 134 (3): 168-180

6. $\mathrm{KIM} \mathrm{CH}$, et al. Safety and efficacy of minimally invasive stereotactic aspiration with multicatheter insertion compared with conventional craniotomy for large spontaneous intracerebral hemorrhage (50 ml). World Neurosurgery, 2019; 128: 787-795.

7. KOENIG MA. Cerebral edema and elevated intracranial pressure. American Academy of Neurology, 2018; 24(6): $1588-1602$.

8. KOLIAS AG, et al. Decompressive craniectomy following traumatic brain injury: developing the evidence base. British Journal of Neurosurgery, 2016; 30(2): 246-250.

9. KOLIAS AG, et al. The current status of decompressive craniectomy in traumatic brain injury. Current Trauma Reports, 2018; 4(4): 326-332.

10. KURLAND DB, et al. Complications associated with decompressive craniectomy: a systematic review. Neurocritical Care Society, 2015; 23(2): 292-304.

11. NAM TM, KIM YZ. A meta-analysis for evaluating efficacy of neuroendoscopic surgery versus craniotomy for supratentorial hypertensive intracerebral hemorrhage. Journal of Cerebrovascular and Endovascular Neurosurgery, 2019; 21(1): 11-17.

12. OLIVEIRA ML, et al. Intracranial hypertension and cerebral autoregulation: a systematic review and meta-analysis. World Neurosurgery, 2018; 113:110-124.

13. QURESHI AI, et al. Intracerebral hemorrhage. The Lancet, 2009; 373(9675): 1632-1644.

14. RAMANAN M, SHANKAR A. Minimally invasive surgery for primary supratentorial intracerebral hemorrhage. J Clin Neurosci, 2013; 20: 1650-1658.

15. RUBIANO AM, et al. The role of decompressive craniectomy in the context of severe Traumatic Brain Injury. Frontiers in Neurology, 2019; 10: 1063.

16. SCAGGIANTE J, et al. Minimally invasive surgery for intracerebral hemorrhage an updated meta-analysis of randomized controlled trials. Stroke, 2018; 49(11): 2612-2620.

17. SMITH M. Refractory intracranial hypertension: the role of decompressive craniectomy. Anesthesia \& Analgesia, 2017; 125: 1999-2008.

18. VESPA PM, et al. Surgical trials in intracerebral hemorrhage. Stroke, 2013; 44: 79-82.

19. WANG GQ, et al. Can minimally invasive puncture and drainage for hypertensive spontaneous basal ganglia intracerebral hemorrhage improve patient outcome: a prospective non-randomized comparative study. Military Med Res. 2014; 1(1): 1-12.

20. WANG W, et al. Minimally invasive surgery for patients with hypertensive intracerebral hemorrhage with large hematoma volume: a retrospective study. World Neurosurgery, 2017; 105: 348-358.

21. XIA Z, et al. Minimally invasive surgery is superior to conventional craniotomy in patients with spontaneous supratentorial intracerebral hemorrhage: a systematic review and meta-analysis. World Neurosurgery, 2018; 115: 266273.

22. $\mathrm{XU} \mathrm{X}$, et al. Effectiveness of endoscopic surgery for supratentorial hypertensive intracerebral hemorrhage: a comparison with craniotomy. Journal of Neurosurgery, 2018; 128: 649.

23. YAMASHIRO S, et al. Effectiveness of endoscopic surgery for comatose patients with large supratentorial intracerebral hemorrhages. Neurol Med Chir, 2015; 55(11): 819-823.

24. YE Z, et al. Comparison of neuroendoscopic surgery and craniotomy for supratentorial hypertensive intracerebral hemorrhage: a meta-analysis. Medicine, 2017; 96: 35.

25. ZHAO XH, et al. Efficacy of neuroendoscopic surgery versus craniotomy for supratentorial hypertensive intracerebral hemorrhage: a meta-analysis of randomized controlled trials. Brain and Behavior, 2019; 9(12): 1471. 